\title{
Response to Correspondence by Fakhr-Movahedi et al.
}

\author{
Muna Bhattarai ${ }^{1} \cdot$ Khomapak Maneewat $^{2} \cdot$ Wipa Sae-Sia $^{2}$
}

Received: 28 February 2018 / Accepted: 5 March 2018 / Published online: 5 April 2018

(c) International Spinal Cord Society 2018

First, we would like to thank the authors Tayyebeh AliAbadi, Mohsen Soleimani, and Ali Fakhr-Movahedi for providing invaluable comments regarding our paper. We believe it will be really helpful for us to take more academic considerations in order to improve our research in the future and strengthen the findings. We hereby respond to all of the concerns point by point.

1. Numerical Rating Scale: There are reasons underpinning our decision not to do the reliability testing of the Numerical Rating Scale (NRS). First, the NRS has been evidenced and recommended as one of the most reliable and universal tools to assess pain in persons who can communicate $[1,2]$. Second, the NRS has been widely used in Nepal and a recent study conducted among Nepalese with musculoskeletal pain yielded good validity, reliability, and responsiveness using the NRS [3]. Third, pain intensity was collected in terms of injury-related information of the participants but not as an outcome measure in our study; reliability testing in our study focused on the main research instrument (Connor-Davidson Resilience Scale (CD-RISC) 25). However, this inquiry raises our awareness of examining the reliability of the instruments when using a new sample. We are very concerned about this point and we actually accept this as one of the limitations of our study.

2. Multivariate versus multiple regression: In our study, we have a single dependent variable (resilience). As the first step, we used simple linear regression (univariate analysis). Then we used multiple regression (multivariate analysis) entering only the factors that were significant in the simple regression [4]. It is

Muna Bhattarai

bhattarai.moona@gmail.com

Hope International College, Satdobato, Lalitpur, Nepal

2 Faculty of Nursing, Prince of Songkla University, Hat Yai, Songkhla, Thailand notable that multiple regression is one kind of multivariate statistics. The term 'multivariate regression' was then used interchangeably with multiple regression [5]; therefore, we used this term in our study. We also believe it would be unequivocal to the readers to use multiple regression in lieu of multivariate regression.

3. Cut-off points of resilience: Since there are no published standard cut-off scores for the CD-RISC, we took the mean resilience score from a previous study as a reference and applied the cut-off scores to the similar context in Nepal since India and Nepal are neighboring countries. Moreover, both of these two countries are eastern and developing countries that are quite similar in a cultural context. In addition, the study by Rajan et al. was conducted among the parents (age range 21-60 years) of children with disabilities rather than the children. In the manual of CD-RISC, both of the samples belong to a group of people who are under stress [6]. Furthermore, participants from both studies were adults and mostly from rural areas. In addition, it may not be helpful to use the score distribution in our own sample to describe proportions of the participants as low or high. Hence, we used the reference from the previous similar study.

We hope the information given will help the readers to better understand each inquiry. We truly appreciate all of the feedback from the readers and we thank the editor for providing the time and concern on this matter.

\section{Compliance with ethical standards}

Conflict of interest The authors declare that they have no conflict of interest. 


\section{References}

1. Castarlenas E, Jensen MP, von Baeyer CL, Miró J. Psychometric properties of the numerical rating scale to assess self-reported pain intensity in children and adolescents: a systematic review. Clin J Pain. 2017;33:376-83.

2. Hawker GA, Mian S, Kendzerska T, French M. Measures of adult pain: Visual Analog Scale for pain (VAS pain), Numeric Rating Scale for pain (NRS pain), McGill Pain Questionnaire (MPQ), Short-Form McGill Pain Questionnaire (SF-MPQ), Chronic Pain Grade Scale (Cpgs), Short Form-36 Bodily Pain Scale (SF-36 BPS), and measure of Intermittent and Constant Osteoarthritis Pain (ICOAP). Arthritis care \& Res. 2011;63(S11):S240-52.
3. Sharma S, Palanchoke J, Reed D, Abbott JH. Translation, crosscultural adaptation and psychometric properties of the Nepali versions of numerical pain rating scale and global rating of change. Health Qual Life Outcomes. 2017;15:236.

4. Bhattarai M, Maneewat K, Sae-Sia W. Determinants of resilience among people who sustained spinal cord injury from the 2015 earthquake in Nepal. Spinal Cord. 2018;56:78-83.

5. Zhou X, Song H, Hu M, Li X, Cai Y, Huang G, et al. Risk factors of severity of post-traumatic stress disorder among survivors with physical disabilities one year after the Wenchuan earthquake. Psychiatry Res. 2015;228:468-74.

6. Davidson JR, Connor KM. Connor-Davidson Resilience Scale (CD-RISC) manual. Unpublished 2017 and partly accessible at www.cd-risc.com 